\title{
A rapid and non-destructive method for spatial-temporal quantification of colonization by Pseudomonas syringae pv. tomato DC3000 in Arabidopsis and tomato
}

\author{
Leonardo Furci ${ }^{1,2,3^{*}}$, David Pascual-Pardo ${ }^{1,2}$ and Jurriaan Ton ${ }^{1,2^{*}}$
}

\begin{abstract}
Background: The bacterial leaf pathogen Pseudomonas syringae pv tomato (Pst) is the most popular model pathogen for plant pathology research. Previous methods to study the plant-Pst interactions rely on destructive quantification of Pst colonisation, which can be labour- and time-consuming and does not allow for spatial-temporal monitoring of the bacterial colonisation. Here, we describe a rapid and non-destructive method to quantify and visualise spatial-temporal colonisation by Pst in intact leaves of Arabidopsis and tomato.
\end{abstract}

Results: The method presented here uses a bioluminescent Pst DC3000 strain that constitutively expresses the luxCDABE operon from Photorhabdus luminescens (Pst::LUX) and requires a common gel documentation (Gel Doc) system with a sensitive CCD/CMOS camera and imaging software (Photoshop or Image J). By capturing bright field and bioluminescence images from Pst::LUX-infected leaves, we imaged the spatiotemporal dynamics of Pst infection. Analysis of bioluminescence from live Pst bacteria over a 5-day time course after spray inoculation of Arabidopsis revealed transition of the bacterial presence from the older leaves to the younger leaves and apical meristem. Colonisation by Pst:LUX bioluminescence was obtained from digital photos by calculating relative bioluminescence values, which is adjusted for bioluminescence intensity and normalised by leaf surface. This method detected statistically significant differences in Pst::LUX colonisation between Arabidopsis genotypes varying in basal resistance, as well as statistically significant reductions in Pst::LUX colonisation by resistance-inducing treatments in both Arabidopsis and tomato. Comparison of relative bioluminescence values to conventional colony counting on selective agar medium revealed a statistically significant correlation, which was reproducible between different Gel Doc systems.

Conclusions: We present a non-destructive method to quantify colonisation by bioluminescent Pst::LUX in plants. Using a common Gel Doc system and imaging software, our method requires less time and labour than conventional methods that are based on destructive sampling of infected leaf material. Furthermore, in contrast to conventional strategies, our method provides additional information about the spatial-temporal patterns of Pst colonisation.

Keywords: Arabidopsis, Tomato, Pseudomonas syringae pv. tomato, Bioluminescence, Non-destructive assay, Spatialtemporal pathogen colonisation

*Correspondence: leonardo.furci@oist.jp; j.ton@sheffield.ac.uk ' School of Biosciences, University of Sheffield, Western Bank, Sheffield, UK

Full list of author information is available at the end of the article

\section{Background}

Pseudomonas syringae is a hemi-biotrophic Gram-negative bacterial species that infects a wide range of plant species [1]. In the early 1990 s, the P. syringae pathovar tomato strain DC3000 (Pst) from tomato was found to original author(s) and the source, provide a link to the Creative Commons licence, and indicate if changes were made. The images or other third party material in this article are included in the article's Creative Commons licence, unless indicated otherwise in a credit line to the material. If material is not included in the article's Creative Commons licence and your intended use is not permitted by statutory regulation or exceeds the permitted use, you will need to obtain permission directly from the copyright holder. To view a copy of this licence, visit http://creativecommons.org/licenses/by/4.0/. The Creative Commons Public Domain Dedication waiver (http://creativeco mmons.org/publicdomain/zero/1.0/) applies to the data made available in this article, unless otherwise stated in a credit line to the data. 
infect various accessions of Arabidopsis thaliana (Arabidopsis), which led to the establishment of the most popular pathosystem in phytopathological research [2]. The systematic study of the Pst-Arabidopsis interaction has yielded crucial discoveries about plant immunity and plant-pathogen interactions, including bacterial virulence [3], resistance (R)-genes and effector-triggered immunity $[4,5]$, perception of microbial associated molecular patterns (MAMPs) [6, 7], basal resistance [8-10], and various forms of acquired resistance [11-14].

Irrespective of the nature of the research, accurate quantification of pathogen colonisation is crucial for the determination of host resistance. For the Pst-Arabidopsis system, the gold standard to quantify bacterial colonisation is based on counting colony forming units (CFUs) on selective agar plates of serial dilutions from homogenised Pst-inoculated leaves $[4,15]$. Although the use of microtiter plates and multichannel pipettes has made this method more efficient [15], the homogenisation of leaf tissue and subsequent dilution plating remains a labour-intensive step that is prone to errors. The availability of transgenic bioluminescent Pst DC3000 strain expressing a stable chromosomal insertion of the lux$C D A B E$ operon from the insect pathogen Photorhabdus luminescens into Pst (henceforth, Pst::LUX) has enabled an alternative method for determining Pst colonization that does not rely on dilution plating [16]. This procedure relies on measuring photon emission by living Pst::LUX cells in excised leaf discs or ground suspensions from infected plants. Nonetheless, the quantification of bioluminescence with a luminometer still relies on destructive sampling and does not provide information about the spatial-temporal colonization by Pst.

In this study, we describe an improved method for the quantification of Pst colonisation in plants, which requires a common gel documentation (Gel Doc) system with a high-sensitivity CCD/CMOS camera that is suitable for quantification of chemiluminescence. There are two main advantages to this method compared to previously described methods of Pst quantification. Firstly, our method is non-destructive, allowing for repeated measures on the same sample/plant over a time-course without having to increase population size. Secondly, the image-based data acquisition from infected leaf tissues captures valuable information about the spatial-temporal patterns of bacterial colonization in intact plants.

\section{Materials and methods}

\section{Plant materials and growth conditions}

Arabidopsis seeds from wild-type (accession Col-0) and hypersusceptible NahG (Col-0; [17]) were stratified in water at $4{ }^{\circ} \mathrm{C}$ in the dark for 4 days. Seeds were then sown in a sand:M3 compost mixture (1:3) and cultivated under short-day conditions for 2.5 weeks (8.5 h light/15.5 h dark, $21{ }^{\circ} \mathrm{C}, 60 \%$ relative humidity, $\sim 125 \mu \mathrm{mol} \mathrm{s}^{-1} \mathrm{~m}^{-1}$ light intensity). A total of 24 plants of each genotype/ treatment combination were grown in three $60 \mathrm{ml}$-pots (8 plants/pot). Tomato seeds (cv. MoneyMaker) were planted in M3 compost and cultivated under long-day conditions for 2 weeks $\left(16 \mathrm{~h}\right.$ light $/ 8 \mathrm{~h}$ dark, $25{ }^{\circ} \mathrm{C}, 60 \%$ relative humidity, $\sim 200 \mu \mathrm{mol} \mathrm{s}^{-1} \mathrm{~m}^{-1}$ light intensity). A total to 15 tomato plants per treatment were grown in individual $100 \mathrm{ml}$-pots. Resistance was induced at 2 days before bacterial inoculation. For induction of resistance, Arabidopsis plants were sprayed with $250 \mu \mathrm{M}$ benzothiadiazole (BTH) as Bion ${ }^{\circledR}$ (Syngenta) suspended in reverseosmosis water, whereas tomato plants were sprayed with $2.5 \mathrm{mM}$ salicylic acid (SA) suspended in reverse-osmosis water.

\section{Bacteria preparation and inoculation}

Before each inoculation, transgenic Pst::LUX bacteria [16] were cultured from a frozen stock $\left(-80{ }^{\circ} \mathrm{C} ; 20 \%\right.$ $\mathrm{v} / \mathrm{v}$ glycerol) of an overnight $(\mathrm{O} / \mathrm{N})$ culture from single colony. One day before inoculation, $1 \mathrm{ml}$ of frozen stock was added to $50 \mathrm{ml}$ of $\mathrm{KB}$ medium (prepared from 1L: 20 g Proteose Peptone; $15 \mathrm{ml}$ glycerol; $1.5 \mathrm{~g} \mathrm{~K}_{2} \mathrm{HPO}_{4}$; $1.5 \mathrm{~g} \mathrm{MgSO}_{4} \cdot 7 \mathrm{H}_{2} \mathrm{O}$; final $\left.\mathrm{pH} 7.2\right)$ containing $50 \mu \mathrm{g} / \mathrm{ml}$ Rifampicin and $50 \mu \mathrm{g} / \mathrm{ml}$ Kanamycin for positive selection of transgenic Pst::LUX cells. Bacteria were grown $\mathrm{O} / \mathrm{N}$ at $28^{\circ} \mathrm{C}$ in a shaking incubator (Grant-Bio; ES-20). $\mathrm{O} / \mathrm{N}$ cultures of Pst::LUX were centrifuged at $3000 \mathrm{rpm}$ for $3 \mathrm{~min}$, after which the pellet was re-suspended in $10 \mathrm{mM} \mathrm{MgSO}_{4}$ to a final density of $0.2 \mathrm{OD}_{600}$, supplemented with $0.01 \% \mathrm{v} / \mathrm{v}$ surfactant (Silwet L-77, Lehle Seeds), and sprayed onto the leaf surface of 2.5-week-old Arabidopsis plants or 2-week-old tomato. Several hours before inoculation and subsequent days after inoculation, plants were kept at $100 \%$ relative humidity $(\mathrm{RH})$ to facilitate infection.

\section{Non-destructive quantification of Pst colonisation by bioluminescence}

At different days post inoculation (dpi), pots were placed in the dark room of a G:BOX Chemi XRQ (GeneSys) Gel Doc system. Bright field images of infected plants were taken, using the G:BOX Chemi XRQ internal LED illumination ( $80 \mathrm{~ms}$ exposure time). After subsequent incubation in complete darkness of at least 1 min., images of bacterial bioluminescence were acquired without moving the pots (90 s. exposure time, maximum iris opening). To assess robustness of the method, an independent Arabidopsis experiment was conducted with an alternative Gel Doc system (ChemiDoc XRS + Imager, BioRad), using $50 \mathrm{~ms}$. exposure time for bright field images and $60 \mathrm{~s}$. exposure time for bioluminescence acquisition. 
The resolution of the images acquired for bioluminescence analysis was $2300 \times 1700$ px for the G:BOX Chemi XRQ Gel Doc system and $3100 \times 2300$ px for the ChemiDoc XRS + Imager, allowing us to image multiple pots at once over a total surface area of $1039 \mathrm{~cm}^{2}$ and $540 \mathrm{~cm}^{2}$, respectively.

Image-based quantification of bioluminescence was carried out using the common imaging software Photoshop CS6 (Adobe). For each plant, the outlines of the leaves were obtained from bright field images, using a combination of the "Magic Wand" and "Lasso" selection tools. These selections were then saved ("Save Selection" function) and transposed onto the bioluminescence images ("Load Selection" function). The bioluminescence signal from infected leaves were obtained from unprocessed bioluminescence image files (exported as TIFF files) using the "Histogram" function in Adobe Photoshop, which quantifies bioluminescence intensity as a function of pixel brightness (ranging from 0, black pixels, to 255 , brightest white pixels) on the basis of the following formula:

$$
\text { Relative bioluminescence }=\frac{\sum_{i=0}^{255} n_{i}(i)}{n_{\text {tot }}}
$$

where $n_{i}$ indicates the number of pixels at each brightness $i$ ( 0 to 255) within the selected area and $n_{\text {tot }}$ indicates the total number of pixels corresponding to plant area, providing a relative bioluminescence metric that corrects for variation in plant size. To further correct for varying background levels, a concentric circle was placed around individual plants $(\sim 2 \times$ rosette diameter $)$. Background levels of luminescence were then calculated in the area outside the selected plant surface area, which was then subtracted from the relative bioluminescence value originating from within the plant surface area. Other opensource image analysis software, such as GNU-based GIMP or Java-based ImageJ, use an identical function to calculate this value. Because name and functionality of proprietary tools differ across software, we detailed the different steps required to replicate the analysis in each of these programs (Additional file 1: Fig. S1). For correlation analysis between non-destructive quantification of Pst colonisation by bioluminescence and destructive quantification by dilution plating, Arabidopsis plants from each genotype/pre-treatment combination were numbered during image quantification, after which leaf material was harvested for colony plating.

\section{Destructive quantification of Pst colonisation by colony plating}

Following image acquisition, each Arabidopsis seedling was weighted and ground in $1.5 \mathrm{ml}$ Eppendorf tubes containing $750 \mu \mathrm{l}$ of ice-cold $10 \mathrm{mM} \mathrm{MgSO}_{4}$, using a pellet pestle. From each homogenised suspension, an aliquote of $50 \mu \mathrm{l}$ was then serial-diluted, using 96-wells microtiter plates $\left(\right.$ Costar $\left.^{\circledR}\right)$ containing $200 \mu \mathrm{l}$ of $10 \mathrm{mM}$ $\mathrm{MgSO}_{4}$. Twelve samples in each plate were serial-diluted eight times (fivefold dilution steps) and plated onto selective $\mathrm{KB}$ agar plates containing $50 \mu \mathrm{g} / \mathrm{ml}$ Rifampicin and $50 \mu \mathrm{g} / \mathrm{ml}$ Kanamycin, using 96-wells Scienceware ${ }^{\circledR}$ replicator (Sigma-Aldrich, plating $10 \mu \mathrm{l}$ per pin). From each well, two technical replicates were plated onto separate plates and incubated at $28{ }^{\circ} \mathrm{C}$ incubator for $36 \mathrm{~h}$ before enumeration of colony forming units (CFUs). For each sample, CFU counts were averaged between two-to-three different serial dilutions and between technical replicates and normalised to plant fresh weight (mg).

\section{Statistical analysis}

One-way analysis of variance (ANOVA) with Tukey's post-hoc test and Student's t-tests were performed using SPSS Statistics (v24.0, IBM). Pearson's correlation analysis was performed using R software (v4.1.0).

\section{Results}

Quantification of in planta bioluminescence by Pst::LUX

The method described here relies on a Gel Doc system fitted with CCD/CMOS camera for detection of chemiluminescence and the transgenic Pst DC3000 strain, which carries a stable chromosomal insertion of the $\operatorname{lux} C D A B E$ operon from $P$. luminescens and is kanamycin-resistant [16]. Following the acquisition of digital bright field images of inoculated plants, the bacterial bioluminescence signal is acquired in complete darkness, without moving the pots. Before acquisition of the bioluminescence signal by the camera, it is important keep plants in complete darkness for at least $1 \mathrm{~min}$. to prevent delayed chlorophyll fluorescence, which is partially within the visible spectrum and can thus be detected by camera as a confounding signal [18]. To prevent variable contrastenhancing modifications by proprietary software of the Gel Doc system, the unprocessed bioluminescence images (TIFF) should be exported before selection and quantification of bioluminescence by imaging software. Bright field and bioluminescence should be of identical pixel size to facilitate the superimposing of the bioluminescence signal onto leaf area from the bright field image. Examples of bright field and bioluminescence images of Arabidopsis are presented in Fig. 1a and Additional file 2: Fig. S2, showing that the bacteria migrate from fully expanded leaves at 2 and 3 dpi to younger leaves in the centre of the rosette by 4 and 5 dpi (Fig. 1a and Additional file 2: Fig. S2). Hence, non-destructive analysis of bioluminescence by Pst::LUX visualises the spatial patterns of in planta colonisation by this pathogen. 

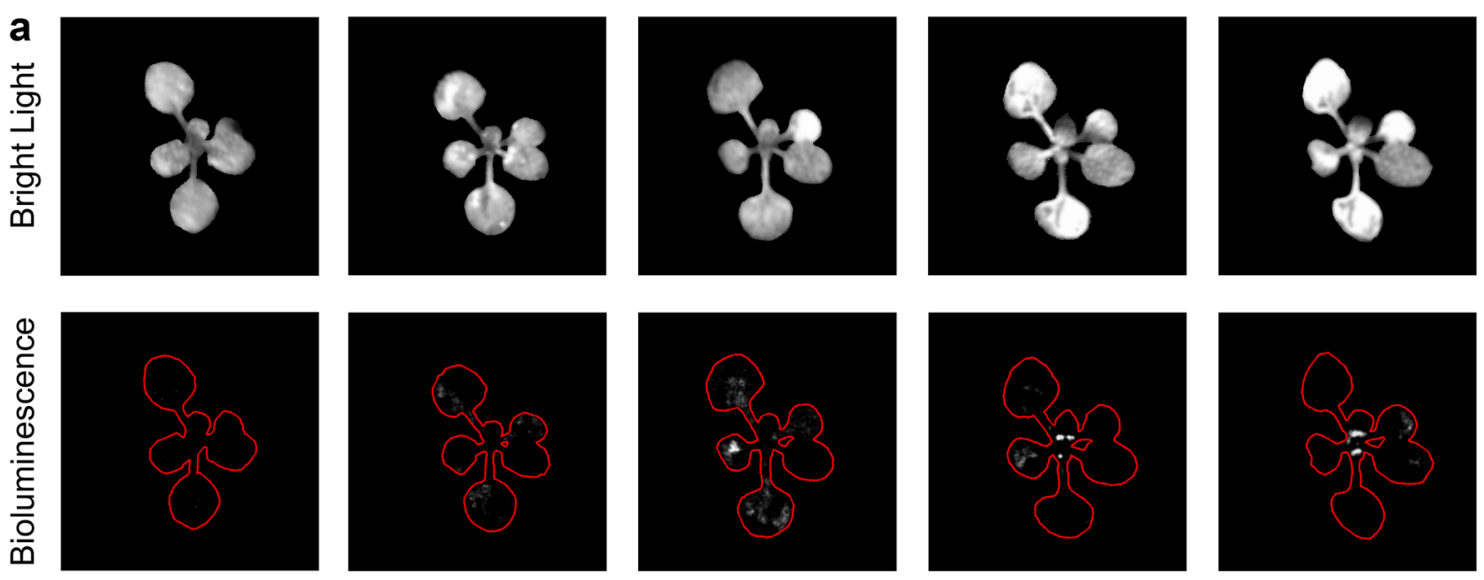

3dpi

$4 d p i$

$5 \mathrm{dpi}$

b

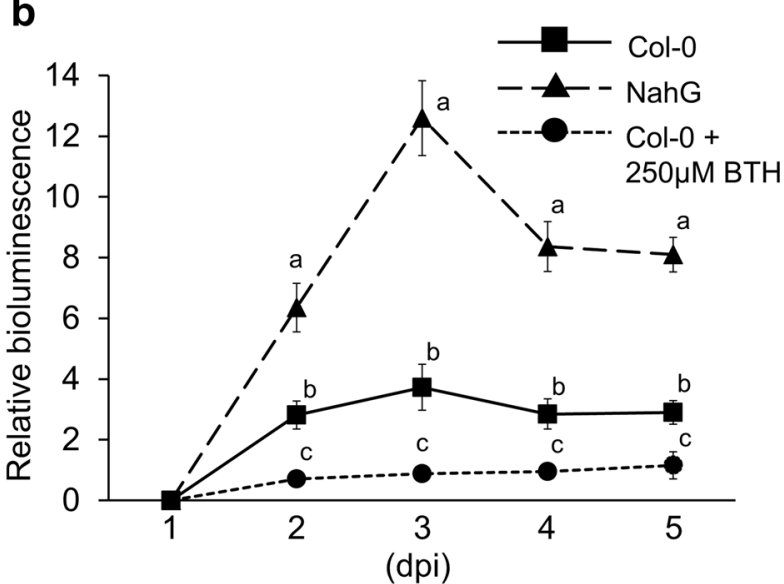

C

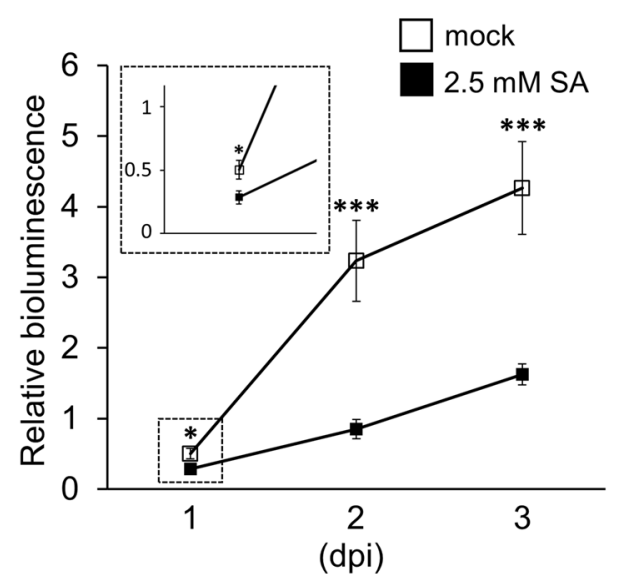

Fig. 1 Non-destructive visualisation and quantification of leaf colonisation by bioluminscent $P$. syringae pv. tomato D3000 expressing the I UXCDABE operon from Photorhabdus luminescens (Pst::LUX). A Representative example of the spatial-temporal pattern Pst::LUX colonisation in a hyper-susceptible NahG plant of Arabidopsis. Top panels show black and white images of the plant taken under bright field illumination. Bottom panels show bacterial bioluminescence acquired from the same plant by a quantum efficiency CCD camera in complete darkness. Red outlines indicate the plant surface area obtained from the bright field images. B Quantification of Pst::LUX colonisation of Arabidopsis plants that vary in salicylic acid (SA)-dependent resistance. Bacterial bioluminescence was measured in hyper-susceptible NahG, wild-type Col-0 and Col-0 pretreated with $250 \mu \mathrm{M}$ of the resistance-inducing SA analogue BTH. Data represent relative bioluminscence values normalised by leaf surface area per plant. Statistically significant differences between genotypes/treatments at each time-point (letters) were assesed by one-way ANOVA, followed by Tukey's post-hoc for multiple comparisons ( $p<0.05$ ). Error bars represent standard errors of the mean $(n=24)$. C Quantification of Pst::LUX colonisation of tomato plants (cv. MoneyMaker) that vary in SA-dependent resistance. Bioluminescence was measured in plants pre-treated with water (mock; susceptible) or $2.5 \mathrm{mM} \mathrm{SA}$ (resistant). Data represent relative bioluminscence values normalised by leaf surface area per plant. Statistically significant differences between treatments at each time-point were assesed by a Student's $t$-test $\left({ }^{*} p<0.05 ;{ }^{* * *} p<0.001\right)$. Error bars represent standard error of the mean $(n=15)$. dpi: days post inoculation

\section{Time-course analysis of Pst::LUX colonisation} in Arabidopsis plants vary in quantitative resistance Using the method described above, we compared Pst::LUX colonisation after inoculation of 17-day-old Arabidopsis plants that vary in quantitative SA-dependent resistance (Fig. 1b). Transgenic NahG plants, which are strongly affected in endogenous accumulation of the defence regulatory hormone SA [17], displayed statistically enhanced levels of bioluminescence compared to Col-0 wild-type plants at all time-points analysed (Fig. 1b, one-way ANOVA). On the other hand, resistance-inducing treatment of Col-0 with $250 \mu \mathrm{M}$ of the functional SA analogue BTH [11] at 2 days before bacterial inoculation resulted in a statistically significant reduction of bioluminescence compared to un-treated Col-0 plants (Fig. 1b; one-way ANOVA). Furthermore, the bioluminescence in hyper-susceptible NahG and untreated susceptible Col-0 peaked at 3 dpi (Fig. 1b) Thus, 
our non-destructive quantification of Pst::LUX colonisation can distinguish differences in SA-dependent resistance over time.

\section{Time-course analysis of Pst::LUX colonisation in tomato}

To examine whether our non-destructive method for quantification of Pst::LUX colonisation can be applied to other plant species than Arabidopsis, we measured bioluminescence in susceptible and resistant tomato plants at different days after inoculation. Since chemical activation of the SA response in tomato induces resistance against Pst [19], we treated leaves of 12-day-old tomato (cv. MoneyMaker) with $2.5 \mathrm{mM}$ SA at 2 days before sprayinoculation with Pst::LUX. Bioluminescence was quantified from 1 to $3 \mathrm{dpi}$. At later time-points, plants started abscising diseased leaves, which prevented further reliable quantification of Pst::LUX colonisation. As shown in Fig. 1c, pre-treatment with SA resulted in a statistically significant reduction of relative bioluminescence compared to control-treated tomato across all time-points (Fig. 1c; Student's T-test). Notably, our method was sufficiently sensitive to detect a small, yet statistically significant, difference as early as $1 \mathrm{dpi}$ (Fig. 1c, inset). Hence, the non-destructive quantification of Pst::LUX colonisation is suitable to distinguish differences in SA-dependent resistance in multiple plant species across different stages of infection.

\section{Comparison of Pst::LUX quantification by bioluminescence and colony plating}

To further validate the effectiveness of our method, we compared the non-destructive quantification of bioluminescence to conventional colony plating of Pst::LUX [15]. Quantification of colonisation by both methods was performed on the same plants at 3dpi after spray-inoculation, which is when bioluminescence peaked in the previous experiment (Fig. 1a, b). As is shown in Fig. 2a both methods detected similar and statistically significant differences in bacterial colonisation between hyper-susceptible NahG, Col-0, and Col-0 expressing BTH-induced resistance (Fig. 2a; one-way ANOVA followed by Tukey's post-hoc test for multiple comparisons). Furthermore, Pearson's correlation analysis between the relative bioluminescence values and fresh weight-normalised CFU counts revealed a positive correlation between both variables, which was statistically significant (Fig. 2b). In a separate experiment, we repeated the comparison between bioluminescence and colony plating for Col-0 and NahG plants with a different Gel Doc system (ChemiDoc XRS + Imager, BioRad), in order to assess robustness of the method with different lab equipment. Using a slightly shorter exposure time $(60 \mathrm{~s}$ for the BioRad Gel Doc versus $90 \mathrm{~s}$ for the G:BOX Gel doc), we

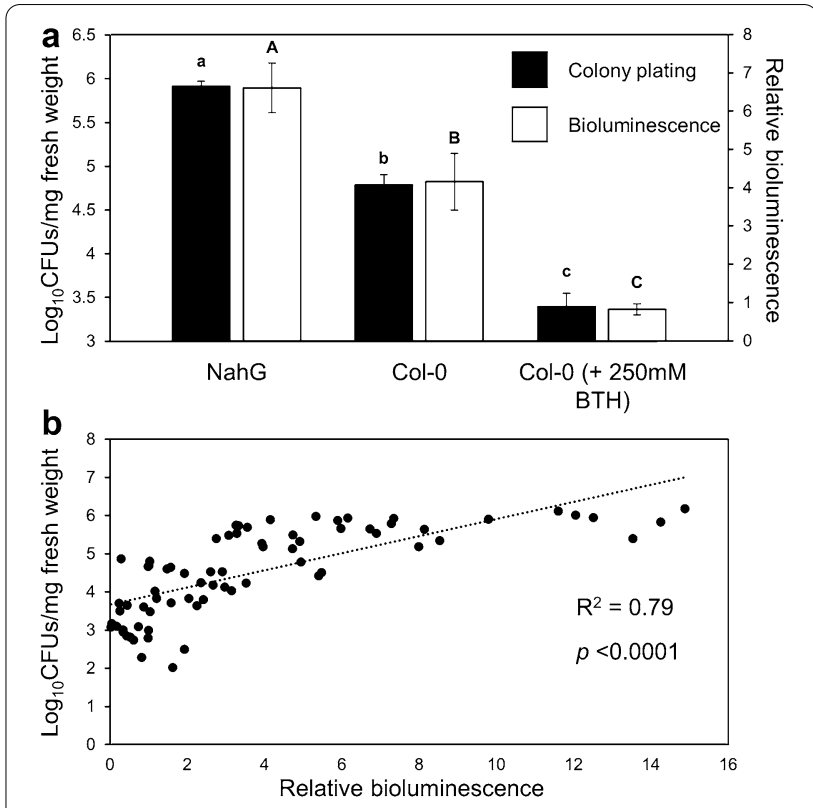

Fig. 2 Comparison between methods for quantification of Pst::LUX colonisation in Arabidopsis plants varying in SA-dependent resistance. A Shown are mean $\log _{10}$-transformed colony forming units (CFU) per milligram of fresh weight (black bars) and mean relative bioluminescence values per plant (white bars) for hyper-susceptible NahG plants, susceptible Col-0 plants and resistant Col-0 plants that had been pretreated with $250 \mu \mathrm{M}$ BTH. Bacterial bioluminescence was measured in planta before samples were harvested for colony plating. Different letters indicate statistically significant differences between genotypes/treatments, using one-way ANOVA, followed by Tukey's post-hoc analysis for multiple comparisons ( $p<0.05$; small letters: plate dilution analysis; capital letters: relative bioluminescence analysis). Error bars represent standard errors of the mean $(n=24)$. B Pearson's correlation analysisb etween CFUs per milligram of plant fresh weight and relative bioluminescence. Dots represent individual samples from all genotypes/treatment combinations presented in $\mathbf{A}$

obtained similar results: a statistically significant difference in relatively bioluminescence between Col-0 and NahG (Additional file 3: Fig. S3a) and a positive correlation between relative bioluminescence and normalised CFU values that was statistically significant (Additional file 3: Fig. S3b, Pearson's correlation, $p=0.0011)$. Based on both experiments, our method could detect variable levels of Pst colonisation at $3 \mathrm{dpi}$, ranging from as low as $\log _{10}(2.02) \mathrm{CFUs} / \mathrm{mg}$ of fresh plant weight (Fig. 2) to as high as $\log _{10}(10.39)$ CFUs/mg of fresh plant weight (Additional file 3: Fig. S3).

\section{Discussion}

Precise quantification of plant colonisation by microbial pathogens is fundamental for mechanistic studies of plant-pathogen interactions. The Pst-Arabidopsis interaction is the most popular pathosystem in 
molecular plant pathology and has largely relied on colony (CFU) counting of infected leaf extracts after dilution plating on selective agar medium. While this method has been optimised for higher throughput [15], the homogenisation of plant tissues and dilution of samples remains a limitation. A previously described method, which measures bioluminescence emission by transgenic Pst::LUX bacteria in excised leaf discs of infected plants, has improved the throughput capacity and generated results that were comparable to colony plating [16]. However, this latter method remains destructive and therefore prevents analysis of the spatial-temporal patterning of pathogen infection, which is relevant for studies of quantitative resistance and pathogen effectors mediating systemic susceptibility. Here, we have introduced a novel non-destructive method that is based on bioluminescence by Pst::LUX, which combines high-throughput quantification with spatial-temporal visualisation of bacterial colonisation.

Our method requires a high-sensitivity CCD/CMOS camera, which are commonly used in Gel Doc systems to quantify chemiluminescence of non-radioactive probes, and common imaging software. For the calculation of relative bioluminescence, we used Adobe Photoshop, but open-source software such as ImageJ and GIMP are equally suitable. The non-destructive nature of our method allows for repeated measurements of the same plants. Consequently, time-course analysis of Pst colonisation is not confounded by inter-plant variation and does not require growing multiple sets of plants for each time-point. As a result, this method requires fewer plants, less controlled environment space, less time and less labour to generate precise time-course analysis of virulence and/or basal resistance in larger populations of plants. We have successfully used our method to quantify Pst::LUX colonisation in large-scale experiments consisting of $>700$ samples in a single batch over a three-day time course, amounting to $2000+$ individual measurements (Furci and Ton, unpublished results). By comparison, Fan et al. [16] reported scoring of bacterial bioluminescence in excised leaf discs consisting of 364 samples [16], whereas high-throughput colony plating is limited to approximately 100-150 samples per batch [15]. The method distinguishes subtle differences in quantitative resistance between plant genotypes or resistance-inducing treatments and yields comparable results to destructive colony plating (Figs. 1 and 2, Additional file 3: Fig. S3). Furthermore, we have successfully employed two commonly used Gel Doc systems (Fig. 2 and Additional file 3: Fig. S3) to quantify Pst::LUX colonisation in two different plant species (Fig. 1), which benchmarks our method for future studies of Pst pathosystems.
The image-based data collection of our method generates information about the spatiotemporal dynamics of Pst colonisation in individual plants (Fig. 1a and Additional file 2: Fig. S2). In Arabidopsis, Yu et al. [20] demonstrated that multiplication and vascular propagation of GFP-expressing Pst DC3000 is resisted through epigenetic mechanisms involving ROS1-dependent DNA demethylation [20]. Furthermore, pathogenic Pseudomonas syringae isolates in other species have been reported to migrate from the initial site of inoculation through the vascular system [21, 22]. While other factors could explain the observed transition in bacterial colonisation from the mature leaves to younger leaves (e.g., a late onset of bacterial infection in the central rosette following spray-inoculation), the results presented in Figs. 1a and Additional file 2: Fig. S2 validate our methodology as a powerful tool to precisely quantify spatial-temporal colonisation of Pst with commonly available equipment. Future experiments involving local Pst::LUX inoculation followed by temporal analysis of relative bioluminescence could shed further lights on the systemic colonisation of Pst and associated defence mechanisms.

This ability to capture spatiotemporal patterning of Pst colonisation in a high-throughput manner creates new opportunities for large-scale genetic screens to identify bacterial effectors that facilitate plant colonisation or plant genes that control quantitative resistance barriers preventing systemic colonisation by the pathogen. Development of fully automated batch-processing pipelines of the bright field and bioluminescence images would further reduce the time required for the image analysis, which will be of particular benefit to large time-course experiments at population-wide scales ( $>1000$ samples). Previous studies have used changes in chlorophyll fluorescence to monitor Pst infection in a non-destructive manner [23]. Combining such non-destructive methods for the quantification of disease symptom development with our method would be a powerful tool to compare the molecular-genetic relationship between tolerance and resistance to Pst. Thus, our method can be linked to other phenotyping platforms, such as chlorophyll fluorescence imaging and hyperspectral imaging, which could drive further innovations in neural-network artificial intelligence software for automated data acquisition, integration and analysis $[24,25]$.

\section{Conclusions}

We have developed a non-destructive method for rapid quantification and spatial-temporal visualisation of Pst colonisation in plants. Our method requires a commonly used Gel Doc system that is equipped with a sensitive $\mathrm{CCD} / \mathrm{CMOS}$ camera for acquisition of chemiluminescence. The possibility of repeated 
measurements of Pst colonisation in the same plants reduces inter-plant variation in time-course analyses. The non-destructive and rapid nature of the method, combined with the image-based data acquisition, enables rapid quantitative screening of the spatiotemporal patterns of Pst colonisation, which opens up new avenues for large-scale genetic screens to identify bacterial virulence effectors and plant resistance barriers.

\section{Supplementary Information}

The online version contains supplementary material available at https://doi. org/10.1186/s13007-021-00826-2.

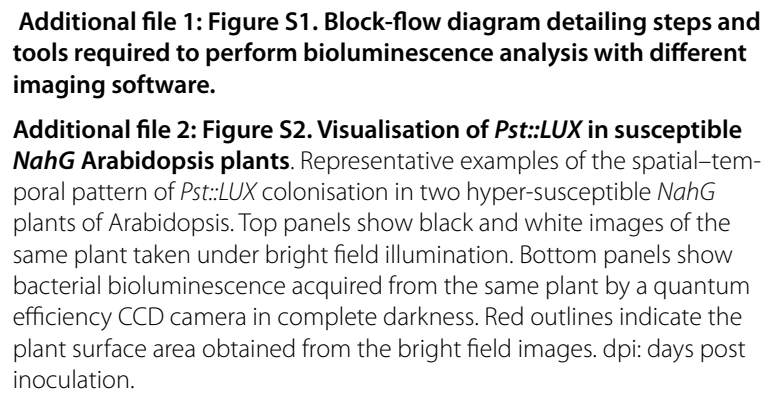

Additional file 3: Figure S3. Comparison between methods for quantification of Pst::LUX colonisation in Arabidopsis genotypes varying in SA-dependent resistance, using a different Gel Doc system A) Shown are mean $\log _{10}$-transformed colony forming units (CFU) per milligram of fresh weight (black bars) and mean relative bioluminescence values per plant (white bars) for hyper-susceptible NahG plants and moderatly susceptible Col-0 plants. Bacterial bioluminescence was measured in planta before samples were harvested for colonoy plating. Asterisks indicate statistically sigificant differences between genotypes (Student's t-test; ***: $p<0.001)$. Error bars represent standard errors of the mean $(n=12$. B) Pearson's correlation analysis between CFUs per milligram of plant fresh weight and relative bioluminescence. Dots represent individual samples from Col-0 and NahG genotypes presented in Fig. S3A.

\section{Acknowledgements}

Not applicable.

\section{Authors' contributions}

JT conceptualized the research and provided resources and supervision. LF and DP performed the experiments and analyzed the data. LF and JT wrote the initial manuscript draft. LF, DP and JT edited the manuscript. All authors read and approved the final manuscript.

\section{Funding}

This work was supported by a grant from the European Research Council (ERC; No. 309944"Prime-A-Plant") to J.T., a Research Leadership Award from the Leverhulme Trust (No. RL-2012-042) to J.T., a BBSRC-IPA Grant to J.T. (BB/ P006698/1) and supplementary grant from Enza Zaden to J.T., and a ERC-PoC Grant to JT (No. 824985 "ChemPrime).

\section{Availability of data and materials}

All raw data analysed in the current study and the Pst::LUX strain can be made available from the corresponding authors on reasonable request.

\section{Declarations}

Ethics approval and consent to participate Not applicable.
Consent for publication

Not applicable.

\section{Competing interests}

All authors declare no conflict of interests about this work.

\section{Author details}

${ }^{1}$ School of Biosciences, University of Sheffield, Western Bank, Sheffield, UK. ${ }^{2}$ P3 Centre for Plant \& Soil Biology, Institute for Sustainable Food, University of Sheffield, Sheffield, UK. ${ }^{3}$ Plant Epigenetics Unit, Okinawa Institute of Science and Technology, Onna-son, Okinawa, Japan.

Received: 2 August 2021 Accepted: 30 November 2021

Published online: 13 December 2021

\section{References}

1. Gardan L, Shafik H, Belouin S, Broch R, Grimont F, Grimont PA. DNA relatedness among the pathovars of Pseudomonas syringae and description of Pseudomonas tremae sp. Nov. and Pseudomonas cannabina sp. Nov. (ex Sutic and Dowson 1959). Int J Syst Bacteriol. 1999;49(Pt 2):469-78.

2. Xin XF, He SY. Pseudomonas syringae pv. tomato DC3000: a model pathogen for probing disease susceptibility and hormone signaling in plants. Annu Rev Phytopathol. 2013;51:473-98.

3. Büttner D, He SY. Type III protein secretion in plant pathogenic bacteria. Plant Physiol. 2009;150(4):1656-64.

4. Whalen MC, Innes RW, Bent AF, Staskawicz BJ. Identification of Pseudomonas syringae pathogens of Arabidopsis and a bacterial locus determining avirulence on both Arabidopsis and soybean. Plant Cell. 1991;3(1):49-59.

5. Dong X, Mindrinos M, Davis KR, Ausubel FM. Induction of Arabidopsis defense genes by virulent and avirulent Pseudomonas syringae strains and by a cloned avirulence gene. Plant Cell. 1991;3(1):61-72.

6. Felix G, Duran JD, Volko S, Boller T. Plants have a sensitive perception system for the most conserved domain of bacterial flagellin. Plant J. 1999;18(3):265-76.

7. Gómez-Gómez L, Boller T. FLS2: an LRR receptor-like kinase involved in the perception of the bacterial elicitor flagellin in Arabidopsis. Mol Cell. 2000;5(6):1003-11.

8. Delaney TP, Uknes S, Vernooij B, Friedrich L, Weymann K, Negrotto D, et al. A central role of salicylic acid in plant disease resistance. Science. 1994;266(5188):1247-50.

9. Glazebrook J. Genes controlling expression of defense responses in Arabidopsis-2001 status. Curr Opin Plant Biol. 2001;4(4):301-8.

10. Katagiri F, Thilmony R, He SY. The Arabidopsis thaliana-pseudomonas syringae interaction. Arabidopsis Book. 2002;1:e0039.

11. Lawton KA, Friedrich L, Hunt M, Weymann K, Delaney T, Kessmann H, et al. Benzothiadiazole induces disease resistance in Arabidopsis by activation of the systemic acquired resistance signal transduction pathway. Plant J. 1996;10(1):71-82.

12. Pieterse CMJ, van Wees SCM, van Pelt JA, Knoester M, Laan R, Gerrits $H$, et al. A novel signaling pathway controlling induced systemic resistance in Arabidopsis. Plant Cell. 1998;10(9):1571-80.

13. Zimmerli L, Jakab G, Metraux JP, Mauch-Mani B. Potentiation of pathogen-specific defense mechanisms in Arabidopsis by beta-aminobutyric acid. Proc Natl Acad Sci USA. 2000;97(23):12920-5.

14 López Sánchez A, Pascual-Pardo D, Furci L, Roberts MR, Ton J. Costs and benefits of transgenerational induced resistance in Arabidopsis. Front Plant Sci. 2021. https://doi.org/10.3389/fpls.2021.644999.

15. Tornero P, Dangl JL. A high-throughput method for quantifying growth of phytopathogenic bacteria in Arabidopsis thaliana. Plant J. 2001;28(4):475-81.

16. Fan J, Crooks C, Lamb C. High-throughput quantitative luminescence assay of the growth in planta of Pseudomonas syringae chromosomally tagged with Photorhabdus luminescens luxCDABE. Plant J. 2008;53(2):393-9.

17. Heck S, Grau T, Buchala A, Métraux J-P, Nawrath C. Genetic evidence that expression of NahG modifies defence pathways independent of salicylic acid biosynthesis in the Arabidopsis-Pseudomonas syringae pv. tomato interaction. Plant J. 2003;36(3):342-52. 
18. Ducruet JM. Chlorophyll thermoluminescence of leaf discs: simple instruments and progress in signal interpretation open the way to new ecophysiological indicators. J Exp Bot. 2003;54(392):2419-30.

19 Herman MAB, Davidson JK, Smart CD. Induction of plant defense gene expression by plant activators and Pseudomonas syringae pv. tomato in greenhouse-grown tomatoes. Phytopathology. 2008;98(11):1226-32.

20. Yu A, Lepère G, Jay F, Wang J, Bapaume L, Wang Y, et al. Dynamics and biological relevance of DNA demethylation in Arabidopsis antibacterial defense. Proc Natl Acad Sci. 2013;110(6):2389-94.

21. Misas-Villamil JC, Kolodziejek I, van der Hoorn RAL. Pseudomonas syringae colonizes distant tissues in Nicotiana benthamiana through xylem vessels. Plant J. 2011;67(5):774-82.

22. Gao X, Huang Q, Zhao Z, Han Q, Ke X, Qin H, et al. Studies on the infection, colonization, and movement of Pseudomonas syringae pv. actinidiae in kiwifruit tissues using a GFPuv-labeled strain. PLOS ONE. 2016;11(3): e0151169.

23. Berger S, Benediktyová Z, Matous K, Bonfig K, Mueller MJ, Nedbal L, et al. Visualization of dynamics of plant-pathogen interaction by novel combination of chlorophyll fluorescence imaging and statistical analysis: differential effects of virulent and avirulent strains of $P$. syringae and of oxylipins on A. thaliana. J Exp Bot. 2007;58(4):797-806.

24. Jiang Y, Li C. Convolutional neural networks for image-based high-throughput plant phenotyping: a review. Plant Phenomics. 2020;2020:4152816

25. Huixian J. The analysis of plants image recognition based on deep learning and artificial neural network. IEEE Access. 2020;8:68828-41.

\section{Publisher's Note}

Springer Nature remains neutral with regard to jurisdictional claims in published maps and institutional affiliations.

- fast, convenient online submission

- thorough peer review by experienced researchers in your field

- rapid publication on acceptance

- support for research data, including large and complex data types

- gold Open Access which fosters wider collaboration and increased citations

- maximum visibility for your research: over $100 \mathrm{M}$ website views per year

At BMC, research is always in progress.

Learn more biomedcentral.com/submissions 